\title{
Sensorless SPMSM Position Estimation Using Position Estimation Error Suppression Control and EKF in Wide Speed Range
}

\author{
Zhanshan Wang, ${ }^{1,2}$ Longhu Quan, ${ }^{1,2}$ and Xiuchong Liu ${ }^{1,2}$ \\ ${ }^{1}$ College of Information Science and Engineering, Northeastern University, Shenyang 110004, China \\ ${ }^{2}$ State Key Laboratory of Synthetical Automation for Process Industries, Northeastern University, Shenyang 110004, China \\ Correspondence should be addressed to Zhanshan Wang; zhanshan_wang@163.com
}

Received 2 April 2014; Accepted 29 June 2014; Published 3 August 2014

Academic Editor: Piermarco Cannarsa

Copyright (C) 2014 Zhanshan Wang et al. This is an open access article distributed under the Creative Commons Attribution License, which permits unrestricted use, distribution, and reproduction in any medium, provided the original work is properly cited.

\begin{abstract}
The control of a high performance alternative current $(\mathrm{AC})$ motor drive under sensorless operation needs the accurate estimation of rotor position. In this paper, one method of accurately estimating rotor position by using both motor complex number model based position estimation and position estimation error suppression proportion integral (PI) controller is proposed for the sensorless control of the surface permanent magnet synchronous motor (SPMSM). In order to guarantee the accuracy of rotor position estimation in the flux-weakening region, one scheme of identifying the permanent magnet flux of SPMSM by extended Kalman filter (EKF) is also proposed, which formed the effective combination method to realize the sensorless control of SPMSM with high accuracy. The simulation results demonstrated the validity and feasibility of the proposed position/speed estimation system.
\end{abstract}

\section{Introduction}

Recently, permanent magnet synchronous motor (PMSM) has been widely used in many automation control fields due to its advantages of superior power density, maintenance-free operation, and high controllability [1-3]. The conventional motor servo control needs a position sensor such as incremental encoder to measure the rotor position and feed it back to the controller for ensuring the accurate control. This sensor increases the cost and size while decreasing the reliability of the drive. Therefore, a position sensorless control for PMSM drive has become popular research direction [4-10].

The primary methods for position estimation under the sensorless condition can be divided into several main categories: adaptive approaches, back-electromagnetic force (back-EMF) based methods, reduced order observer methods, EKF based methods, and signal injection methods for low speed range [3-10]. In these studies, the estimation result is very dependent on the accuracy of system parameters that include the motor parameters like inductance, resistance, and magnetic flux and the unmodeled parameters like inverter dead-time effect and inductance cross-saturation. However, the system parameters vary continuously during the motor dynamic, so the estimation result may be biased or cause the vibration.

In order to realize the accurate position estimation, estimation of rotor position and identifying of system parameters must be employed simultaneously. Reference [11] presents a sensorless position estimation method using the combination of identifying the stator resistance by EKF and estimating the rotor position by back-EMF. References [12-15] present a sensorless position estimation method using the combination of identifying the motor parameters by EKF and estimating the rotor angular speed by model reference adaptive system (MRAS), in which EKF has become one of the most effective ways to identify the parameters of nonlinear system like AC motor $[16,17]$. These studies are concentrated to improve the estimation accuracy of rotor position by identifying one or two of the key parameters like inductance, resistance, and flux. However, because of unmodeled nonlinearities, for example, inverter dead-time effect, inductance crosssaturation, and other motor parameters drift and variation, 
there will be the uncertain phenomena in a close-loop sensorless control with estimated position and speed.

Now, in order to eliminate the influence of system uncertainty that affects the control result, the artificial intelligent techniques such as ANN were applied to estimate the position and speed of sensorless AC motor $[18,19]$. These methods can increase the robustness of the position and speed estimation through making a nonlinear function between the input and output of the control system. But they require the relative long operation time for the real-time process in an actual control system. Many researchers tried to reduce the control error that resulted from the system uncertainty through using the complex number analysis of a control system [20-22]. By the use of the instantaneous power that separated into the real part and imaginary part by the complex number analysis, a rotor position estimation method unrelated to the actual speed and flux linkage in a rated speed area was studied in [22]. In [22], the system parameters used in the position estimation were reduced to the phase inductance and resistance in comparison to the conventional methods. This approach not only is simple in the system analysis, but also can easily separate the system variables into the real part and the error (imaginary) part caused by the system uncertainty. In this paper, a novel method that eliminates the influence of entire system uncertainty in the position estimation of sensorless SPMSM is realized by the motor complex number model. However, severe demagnetization in the flux-weakening region can break estimating the position accurately and can also cause the instability problem in the whole control system [10]. The flux of motor is following a Gaussian distribution in the flux-weakening region [1]. References $[5,23]$ revealed that EKF is useful in identifying the accidental variables that are following the Gaussian distribution. In the previous studies, identifying the permanent magnet flux by EKF has focused on the interior permanent magnet synchronous motor (IPMSM) and asynchronous motor, but SPMSM has not been studied.

This paper presents a novel robust position estimation method in which its dynamic position estimation performance is not affected by system parameter variations. The rotor position angle is obtained by a novel position estimator over the wide speed range including low speed region and flux-weakening region. First, the mathematical model of position estimator composed of the real value (real part) and error value (imaginary part) of the estimated rotor position angle is presented. Second, in order to suppress the position estimation error to zero, a position type PI controller is applied to imaginary part of the proposed position estimator. The proposed method can enhance the accuracy and robustness of position estimation by suppressing to zero the estimation error that may be caused by the uncertainty of system parameters in a rated speed range including the low speed region. Third, in order to expand the robustness to the fluxweakening region, the permanent magnet flux identification model of SPMSM by EKF is proposed; the affection that is caused by uncertainty of permanent magnet flux during the motor at the flux-weakening region is eliminated.

The paper is organized as follows. The mathematical model of SPMSM and the principle of the proposed position estimation scheme are shown in Section 2. The combination of a complex number model based position estimator and an error suppression PI control is given in Section 3. An online identification scheme of permanent magnet flux based on EKF is presented in Section 4. The SPMSM vector control system and detailed control scheme are given in Section 5. The simulation block and simulation results are presented in Section 6, which verify the effectiveness of the proposed control method.

\section{Basic Control Equations of SPMSM and Novel Sensorless Control Scheme}

2.1. Basic Control Equations of SPMSM. In the paper, the vector control of SPMSM is considered in a rotor-fixed frame (d-q frame) $[8,10]$ as follows:

$$
\begin{aligned}
& u_{d}=R_{S} i_{d}+L_{S} \frac{d}{d t} i_{d}-L_{S} i_{q} \omega_{k} \\
& u_{q}=R_{S} i_{q}+L_{S} \frac{d}{d t} i_{q}+L_{S} i_{d} \omega_{k}+\psi_{m} \omega_{k} \\
& \psi_{d}=L_{S} i_{d}+\psi_{m} \\
& \psi_{q}=L_{S} i_{q}
\end{aligned}
$$

where $u_{d}, u_{q}, i_{d}$, and $i_{q}$ are $d$-q components of the voltage and current in stator and $\omega_{k}$ is an electrical angle speed of rotor in $d-q$ frame. $R_{S}$ and $L_{S}$ are the resistance and inductance of stator, respectively. $\psi_{m}$ is a rotor permanent magnet flux; $\psi_{d}$ and $\psi_{q}$ are the $d-q$ components of the actual air gap flux of motor.

The electrical angle speed of rotor is

$$
\omega_{k}=\frac{d \theta_{k}}{d t}
$$

where $\theta_{k}$ is the field angle between the $d$ axis of rotor-fixed frame and the $\alpha$ axis of stator-fixed frame ( $\alpha$ - $\beta$ frame) $[8,10]$.

Considering the mechanical load, the dynamic equation of SPMSM drive system is obtained by

$$
J \frac{d}{d t} \omega_{k}=T_{e}-T_{L}-B \omega_{k},
$$

where $T_{e}$ is an electromagnetic torque, $T_{L}$ is a load torque, $J$ is an inertia moment, and $B$ is a viscous damping coefficient.

The electromagnetic torque of motor is given as follows:

$$
T_{e}=p \psi_{m} i_{q}
$$

where $p$ is the number of pole pairs.

In the SPMSM vector control, $d T_{L} / d \omega_{k}>0$ is supposed for all practical speeds [8]. The mentioned system is usually used in simulations as well as in design of the current controller and speed controller. In SPMSM vector control of this paper, the $d$ axis current reference is controlled to be zero and the motor torque is controlled by the $q$ axis current. 


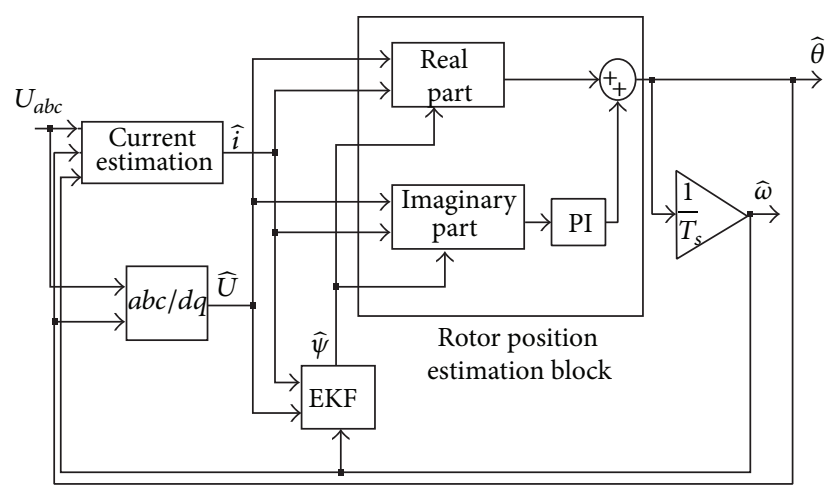

FIGURE 1: Block diagram of proposed estimation scheme.

2.2. Novel Sensorless Estimation Scheme. The proposed novel position estimation scheme is shown in Figure 1. $U_{\mathrm{abc}}, \hat{i}$, and $\Delta \hat{i}$ refer to a three-phase stator voltage of SPMSM, an estimated current, and an error between command current and estimated current; $\widehat{U}$ and $\widehat{\psi}$ refer to an output of current controller and an identified flux obtained from EKF; $\hat{\theta}$ and $\widehat{\omega}$ refer to an estimated rotor position angle and an estimated rotor angular speed.

In the previous studies [24-28], the "rotor position estimation block" calculated the position estimation value directly using $\hat{i}, \widehat{U}$, and motor parameters without considering the estimation error. The estimation result was too dependent on the accuracy of system parameters. The system parameters contain a certain degree of uncertainty, so the estimation result could not be well. In Figure 1, the "rotor position estimation block" is composed of the real position estimation value and the position estimation error that can be caused by the uncertainty of system parameters. In order to realize this separation, we have derived the SPMSM complex number model based position estimator that can easily separate the real value and error value of position estimation. The estimation result is composed of the sum of real position valve ("real part") and estimation error value ("imaginary part"). The position estimation error is controlled to be zero by the error suppression PI controller. As a result, the accurate estimation result may be obtained. By identifying the permanent magnet flux by EKF, the affection accepted by the magnetic flux change in the flux-weakening region is eliminated.

\section{Complex Number Model of SPMSM and Proposed Position Estimation Scheme}

Stator voltage in the stator-fixed frame ( $\alpha-\beta$ frame) [1] is defined as

$$
U_{S}=R_{S} i_{S}+\frac{\psi_{S}}{d t}
$$

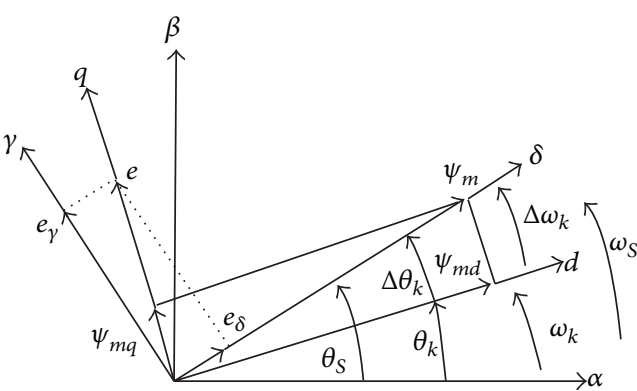

FIGURE 2: Reference frame diagram of SPMSM.

where

$$
\begin{gathered}
U_{S}=U_{\alpha}+j U_{\beta}, \\
i_{S}=i_{\alpha}+j i_{\beta}, \\
\psi_{S}=\psi_{\alpha}+j \psi_{\beta},
\end{gathered}
$$

where $R_{S}$ is a stator resistance. $\psi_{S}, U_{S}$, and $i_{S}$ are rotor flux vector, stator voltage vector, and stator current vector in the $\alpha-\beta$ frame, respectively.

Now, the $\alpha-\beta$ frame rotates with an angular speed $\omega_{k}$, and all quantities can be transformed from the stator-fixed frame to the rotor-fixed frame ( $d-q$ frame) [1]; the results are given as follows:

$$
\begin{gathered}
U_{S}=U_{k} e^{j \theta_{K}}, \quad i_{S}=i_{k} e^{j \theta_{K}}, \quad \psi_{S}=\psi_{k} e^{j \theta_{K}}, \\
\frac{d \psi_{S}}{d t}=\frac{d \psi_{k}}{d t} e^{j \theta_{K}}+j \omega_{k} \psi_{k} e^{j \theta_{K}},
\end{gathered}
$$

where $\psi_{k}, U_{k}$, and $i_{k}$ are rotor flux vector, stator voltage vector, and stator current vector in the $d-q$ frame, respectively. By substituting (8) into (6), the equation of stator voltage in the $d-q$ frame can be expressed as

$$
U_{k}=R_{S} i_{k}+\frac{d \psi_{k}}{d t}+j \omega_{k} \psi_{k}
$$

Equation (9) is usually used for a high performance SPMSM drive. However, this $d-q$ frame cannot be utilized directly in the position sensorless system because the model does not contain any information about the rotor position error. It is necessary to use another reference frame, noted as a $\delta$ - $\gamma$ frame, which lags by position estimation error $\Delta \theta_{k}$ from the $d$-q frame as shown in Figure 2. $\Delta \theta_{k}$ is not a constant and varies continuously during the motor dynamic. The SPMSM model in the $\delta-\gamma$ frame can be derived by transforming the voltage equation (9) of $d-q$ frame into the $\delta-\gamma$ frame as follows:

$$
U_{m}=R_{S} i_{m}+\frac{d \psi_{m}}{d t}+j \omega_{S} \psi_{m}
$$

where $\omega_{S}$ is a rotor electrical angle speed in the $\delta-\gamma$ frame. $\omega_{S}$ is a sum of the actual electrical angle speed $\omega_{k}$ and speed estimation error $\Delta \omega_{k} \cdot \psi_{m}, U_{m}$, and $i_{m}$ are rotor flux vector, stator voltage vector, and stator current vector in the $\delta-\gamma$ 
frame, respectively. If the sensorless control is correct, $\theta_{S}$ of $\delta-\gamma$ frame is convergent to $\theta_{k}$ of $d-q$ frame, the position error $\Delta \theta_{k}$ is also convergent to zero, and all electromagnetic parameters of $\delta$ - $\gamma$ frame can be processed on the $d-q$ frame. In order to simplify consideration of the position estimation, the position error $\Delta \theta_{k}$ is ignored in $\delta-\gamma$ frame and the SPMSM model in $d-q$ frame is employed to estimate the rotor position in position sensorless SPMSM drive system.

Equation (10) can be written as

$$
U_{k}=R_{S} i_{k}+L_{S} \frac{d i_{k}}{d t}+j \omega_{k} \psi_{m}\left(\exp j\left(\Delta \theta_{k}\right)\right)
$$

Using the Euler formula, we can eliminate $j$ from (11):

$$
U_{k}=R_{S} i_{k}+L_{S} \frac{d i_{k}}{d t}+\frac{d \theta_{k}}{d t} \psi_{m}\left(\exp j\left(\Delta \theta_{k}+\frac{\pi}{2}\right)\right) .
$$

We can compute the differential of position $\theta_{k}$ from (12):

$$
\frac{d \theta_{k}}{d t}=\frac{1}{\psi_{m}} \cdot\left(U_{k}-R_{S} i_{k}-L_{S} \frac{d i_{k}}{d t}\right) \cdot \exp \left(-j\left(\Delta \theta_{k}+\frac{\pi}{2}\right)\right) .
$$

The position estimation equation can be obtained from Figure 1 and (13) as follows:

$$
\frac{d \widehat{\theta}_{k}}{d t}=\frac{1}{\psi_{m}} \cdot\left(\widehat{U}_{k}-R_{S} \widehat{i}_{k}-L_{S} \frac{d \hat{i}_{k}}{d t}\right) \cdot \exp \left(-j\left(\Delta \widehat{\theta}_{k}+\frac{\pi}{2}\right)\right),
$$

where the $\widehat{U}_{k}, \hat{i}_{k}$, and $\Delta \widehat{\theta}_{k}$ of (14) correspond to the $\widehat{U}, \hat{i}$, and $\Delta \hat{\theta}$ of Figure 1. Index $k$ means that the parameters in estimation equation are values of $d-q$ frame.

In order to simplify the consideration, let each term of (14) be as follows:

$$
\begin{gathered}
\widehat{U}_{k}-R_{S} \hat{i}_{k}-L_{S} \frac{d \hat{i}_{k}}{d t}=v, \\
\exp \left(-j\left(\Delta \widehat{\theta}_{k}+\frac{\pi}{2}\right)\right)=b .
\end{gathered}
$$

Rotor position estimation equation (14) can be written by the modulus $(v \cdot b)$ as follows:

$$
\frac{d \widehat{\theta}_{k}}{d t}=\frac{1}{\psi_{m}}(v \cdot b) .
$$

For practical implementation, it is necessary to obtain the algorithm in discrete form. Equation (16) can be solved as follows:

$$
\begin{aligned}
\widehat{\theta}_{k}(n)= & \widehat{\theta}_{k}(n-1)+\frac{T}{\psi_{m}}(v(n) \cdot b(n)) \\
= & \widehat{\theta}_{k}(n-1) \\
& +\frac{T}{\psi_{m}}\left\{\widehat{U}_{k}(n)-R_{S} \hat{i}_{k}(n)\right. \\
& \left.-L_{S}\left[\frac{\hat{i}_{k}(n)-\widehat{i}_{k}(n-1)}{T}\right]\right\} \\
& \cdot \exp \left\{-j\left[\Delta \widehat{\theta}_{k}(n)+\frac{\pi}{2}\right]\right\} \\
= & \widehat{\theta}_{k}(n-1) \\
& +\frac{T}{\psi_{m}}\left\{\left[v_{d}(n)+j v_{q}(n)\right]\right. \\
& \left.\times\left[-\sin \left(\Delta \widehat{\theta}_{k}(n)\right)-j \cos \left(\Delta \widehat{\theta}_{k}(n)\right)\right]\right\} \\
= & \widehat{\theta}_{k}(n-1)+\frac{T}{\psi_{m}}\{\widehat{\operatorname{Re}}[v(n) b(n)] \\
& \quad+\widehat{\operatorname{Im}}[v(n) b(n)]\} \\
= & \widehat{\theta}_{k}(n-1)+\Delta \widehat{\theta}_{k}(n) .
\end{aligned}
$$

Now, the estimation $\widehat{\theta}_{k}(n)$ of the actual rotor position $\theta_{k}(n)$ is determined by adding the estimated angle increment $\Delta \widehat{\theta}_{k}(n)$ in one sampling period to the previous estimation $\widehat{\theta}_{k}(n-$ $1)$. There are the real part and imaginary part in the angle increment. Real part is the actual increment of estimation, and the imaginary part is the estimation error. If there are no system parameter errors and the rotor position angle is correctly obtained, the imaginary part of $(v \cdot b)$ is zero. Because the system parameters vary continuously during the motor operation, the position estimation error exists in a certain degree. In order to suppress the position estimation error to zero, the proposed estimation method uses a position type PI controller to the imaginary part of position estimation equation.

The estimated angle increment is obtained as follows:

$$
\begin{aligned}
\Delta \widehat{\theta}_{k}(n)= & \frac{T}{\psi_{m}}\{\operatorname{Re}[v(n) \cdot b(n)] \\
+ & +K_{p}\left[\left(1-\frac{K_{i}}{K_{p}}\right) \operatorname{Im}(v(n) b(n))\right. \\
& \quad-\operatorname{Im}(v(n-1) b(n-1))] \\
& \left.+\Delta \theta_{\text {corr }}(n-1)\right\},
\end{aligned}
$$


where $T, K_{p}$, and $K_{i}$ are sample time of estimation algorithm, proportional gain of PI controller, and $T / T_{i}$, respectively. $T_{i}$ is an integral time constant. The second term of (18) carries out the function of PI control so that the imaginary part of (17) converges to 0 . The position estimation error that may be caused by the uncertainty of system parameters is eliminated through this control.

The estimated angular speed increment $\Delta \widehat{\omega}_{k}(n)$ of the rotation speed is obtained by differentiating estimated position angle increment $\Delta \widehat{\theta}_{k}(n)$ during the sample time $T$. The estimated speed $\widehat{\omega}_{k}(n)$ of the rotor is obtained by

$$
\begin{aligned}
\widehat{\omega}_{k}(n) & =\widehat{\omega}_{k}(n-1)+\Delta \widehat{\omega}_{k}(n) \\
& =\widehat{\omega}_{k}(n-1)+\frac{d \Delta \widehat{\theta}_{k}(n)}{d t} .
\end{aligned}
$$

The imaginary part of (18) must be multiplied by the sign of the estimated speed because the angle between $v$ and $b$ is $\pi$ when speed is negative direction.

In this part, one method of improving the position estimation accuracy and robustness is studied about the sensorless SPMSM vector control. The position estimation error caused by the uncertainty of system parameters could be regulated to zero by this method. The real value and error value of rotor position estimation are correctly separated from the position estimator based on the complex number model of SPMSM. The position estimation error is removed by error suppression PI operation. Using this estimation method, not only is the dynamic estimation of the position and speed fast but also convergence is well, and then the proper estimation accuracy can be obtained.

The position estimation method derived in this part is robust on the uncertainty of system parameters in the rated speed range including low speed. However, severe demagnetization in the flux-weakening area can break estimating the position accurately and can cause the instability problem in the whole control system. The permanent magnet flux $\psi_{m}$ was processed as a constant in position estimation equation (17). In the next part, we present one method that obtains the identified flux $\widehat{\psi}_{m}$ of $\psi_{m}$ by using EKF, in order to guarantee robustness and estimation accuracy in the flux-weakening area.

\section{Online Identification of Permanent Magnet Flux Based on EKF}

4.1. Introduction to EKF. EKF presents the general problem of trying to estimate the states $x \in R^{n}$ that is dominated by the nonlinear stochastic difference equation as follows:

$$
x_{k}=f\left(x_{k-1}, u_{k}, \sigma_{k-1}\right) \text {, }
$$

and the measurement output $y \in R^{n}$ is as follows:

$$
y_{k}=h\left(x_{k}, \rho_{k}\right) \text {, }
$$

where $u_{k}$ is the control vector, the random variables $\sigma_{k-1}$ and $\rho_{k}$ represent the process and measurement noise, and the covariance matrices of these noises are defined as

$$
\begin{aligned}
& Q=E\left[\sigma \cdot \sigma^{T}\right], \\
& R=E\left[\rho \cdot \rho^{T}\right] .
\end{aligned}
$$

The solutions to EKF can be divided into two groups.

(1) Time update equation (predict), which uses the current state and error covariance to obtain the a priori estimations for the next time step.

(i) Project the state ahead:

$$
\widehat{x}_{k}^{-}=f\left(\widehat{x}_{k-1}, u_{k}, 0\right) .
$$

(ii) Project the error covariance ahead:

$$
P_{k}^{-}=A_{k} P_{k-1} A_{k}^{T}+W_{k} Q_{k-1} W_{k}^{T} .
$$

(2) Measurement update equation (correct), which incorporates new measurements into the a priori estimations to obtain improved a posteriori estimations.

(i) Compute EKF gain:

$$
K_{k}=P_{k}^{-} H_{k}^{T}\left(H_{k} P_{k}^{-} H_{k}^{T}+V_{k} R V_{k}^{T}\right)^{-1} .
$$

(ii) Update estimation with measurement:

$$
\widehat{x}_{k}=\widehat{x}_{k}^{-}+K_{k}\left(y_{k}-h\left(\widehat{x}_{k}^{-}, 0\right)\right) .
$$

(iii) Update the error covariance:

$$
P_{k}=\left(1-K_{k} H_{k}\right) P_{k}^{-},
$$

where $\widehat{x}_{k}^{-}$is a priori state estimation at step $k$ given knowledge of the process prior to step $k, \widehat{x}_{k}$ is a posteriori state estimation at step $k$ given measurement $y_{k}$, and $P_{k}^{-}$and $P_{k}$ are the covariance of the a priori and a posteriori estimation errors.

$A_{k}$ is a Jacobian matrix of partial derivatives of $f$ with respect to $x ; A_{k[i, j]}=\left(\partial f_{[i]} / \partial x_{[j]}\right)\left(\widehat{x}_{k-1}, u_{k}, 0\right)$.

$W_{k}$ is a Jacobian matrix of partial derivatives of $f$ with respect to $\sigma ; W_{k[i, j]}=\left(\partial f_{[i]} / \partial \sigma_{[j]}\right)\left(\widehat{x}_{k-1}, u_{k}, 0\right)$.

$H_{k}$ is a Jacobian matrix of partial derivatives of $h$ with respect to $x ; H_{k[i, j]}=\left(\partial h_{[i]} / \partial x_{[j]}\right)\left(\widehat{x}_{k}, 0\right)$.

$V_{k}$ is a Jacobian matrix of partial derivatives of $h$ with respect to $\rho ; V_{k[i, j]}=\left(\partial h_{[i]} / \partial \rho_{[j]}\right)\left(\widehat{x}_{k}, 0\right)$.

4.2. Permanent Magnet Flux Identification of SPMSM Based on EKF. Here, we present one method that identifies the magnetic flux change that can be generated during the motor dynamic. In Section 3 of this paper, the complex number model of SPMSM is used for estimating the accurate position. In (17), the imaginary part of position estimator caused by the uncertainty of motor parameters is controlled to be zero by 
SPMSM sensorless vector control

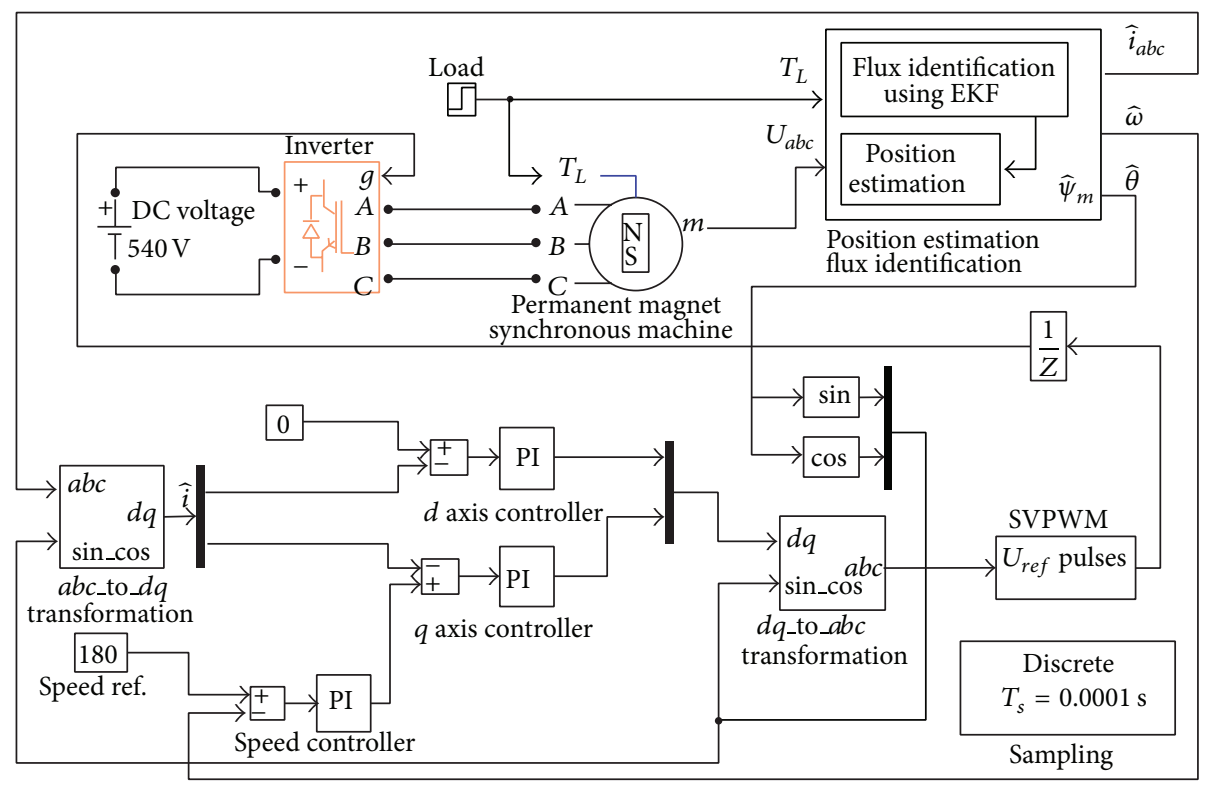

FIGURE 3: Simulation block of sensorless SPMSM drive system by using proposed position/speed estimation and flux identification.

the error suppression PI controller. But, the demagnetization amount must not be controlled to be zero in the fluxweakening area. Calculating the demagnetization amount accurately is one of the most important problems to realize the accurate position estimation and vector control in the flux-weakening area. The complex number model composed of real part and imaginary part may cause the complexity in the flux identification. Therefore, the rotor flux is identified through the combination of SPMSM model (1) and EKF in this paper. Equation (1) with considering the magnetic flux change can be rewritten as follows:

$$
\begin{aligned}
& \frac{d}{d t} \widehat{i}_{d}=-\frac{R_{S} \hat{i}_{d}}{L_{S}}+\widehat{i}_{q} \widehat{\omega}_{k}+\widehat{\omega}_{k} \frac{\widehat{\psi}_{m q}}{L_{S}}+\frac{\widehat{u}_{d}}{L_{S}} \\
& \frac{d}{d t} \hat{i}_{q}=-\frac{R_{S} \hat{i}_{q}}{L_{S}}-\widehat{i}_{d} \widehat{\omega}_{k}-\widehat{\omega}_{k} \frac{\widehat{\psi}_{m d}}{L_{S}}+\frac{\widehat{u}_{q}}{L_{S}} .
\end{aligned}
$$

In order to estimate the magnetic flux, the magnetic flux is chosen as one state variable. Because the magnetic flux linkage cannot change sharply during the motor dynamic [1], its differential is assumed to be zero:

$$
\begin{aligned}
& \frac{d \widehat{\psi}_{m d}}{d t}=0, \\
& \frac{d \widehat{\psi}_{m q}}{d t}=0 .
\end{aligned}
$$

By choosing set of the electric current variables and magnetic flux of the $q$ axis as the state variables, a fourthorder EKF is constructed for identifying the magnetic flux. The state, input, and output vectors are described as follows:

$$
\begin{aligned}
& x=\left[\begin{array}{llll}
\hat{i}_{d} & \widehat{i}_{q} & \widehat{\psi}_{m d} & \widehat{\psi}_{m q}
\end{array}\right]^{T}, \\
& u=\left[\begin{array}{ll}
\frac{\widehat{u}_{d}}{L_{S}} & \frac{\widehat{u}_{q}}{L_{S}}
\end{array}\right]^{T}, \\
& y=\left[\begin{array}{ll}
\hat{i}_{d} & \hat{i}_{q}
\end{array}\right]^{T} \text {. }
\end{aligned}
$$

The system and output measurements are

$$
\begin{gathered}
\frac{d}{d t} x=\left(\begin{array}{cccc}
-\frac{R_{S}}{L_{S}} & \widehat{\omega}_{k} & 0 & \frac{\widehat{\omega}_{k}}{L_{S}} \\
-\widehat{\omega}_{k} & -\frac{R_{S}}{L_{S}} & -\frac{\widehat{\omega}_{k}}{L_{S}} & 0 \\
0 & 0 & 0 & 0 \\
0 & 0 & 0 & 0
\end{array}\right) x+\left(\begin{array}{ll}
1 & 0 \\
0 & 1 \\
0 & 0 \\
0 & 0
\end{array}\right) u, \\
y=\left(\begin{array}{llll}
1 & 0 & 0 & 0 \\
0 & 1 & 0 & 0
\end{array}\right) x .
\end{gathered}
$$

In order to simplify the description, (31) and (32) can be rewritten, respectively, as follows:

$$
\begin{gathered}
\frac{d}{d t} x=L x+M u, \\
y=h x .
\end{gathered}
$$




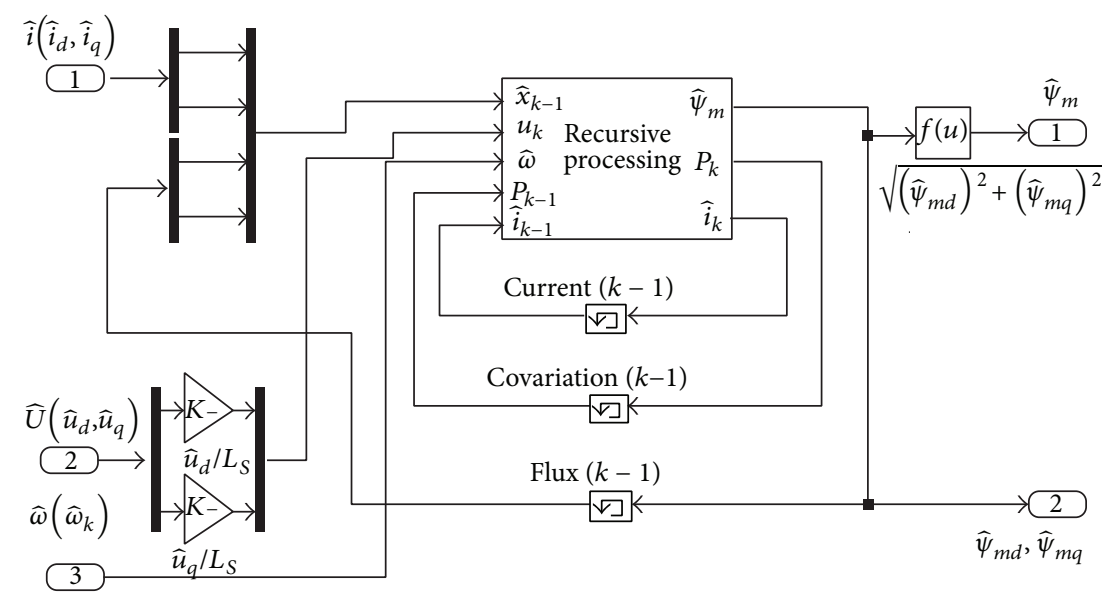

FIGURE 4: Simulation block of identifying PM flux by EKF.

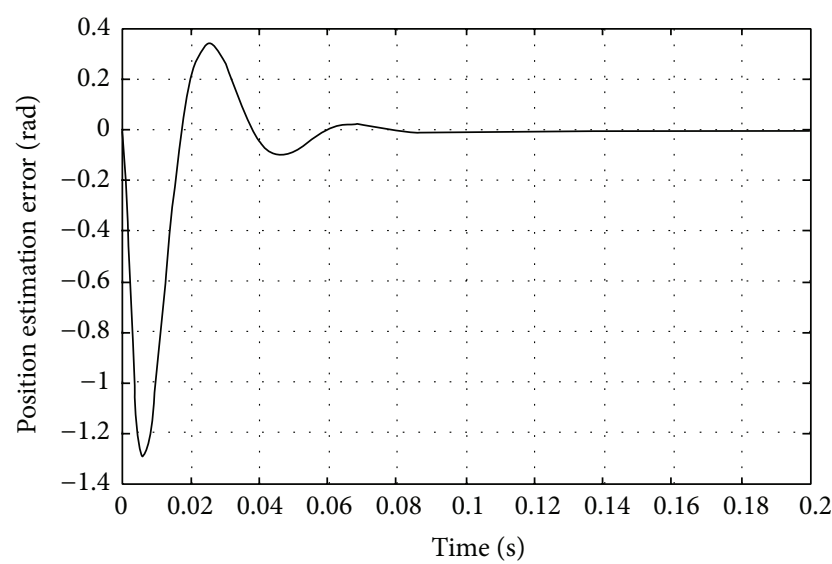

FIGURE 5: Error between actual position and estimated position.

Considering the noise in process and measurement, approximate discrete equations (33) and (34) can be expanded as

$$
\begin{gathered}
x_{k}=(I+L T) x_{k-1}+M T u_{k-1}+\sigma_{k-1}, \\
y_{k}=h x_{k}+\rho_{k},
\end{gathered}
$$

where $I$ is a unity matrix and $T$ is a sample time. Equations (35) and (36) are equivalent to (20) and (21). Therefore, using (35) and (36) can realize (23)-(27) of the EKF recursive processing. The identified flux obtained through EKF is $\widehat{\psi}_{m d}$ and $\widehat{\psi}_{m q}$. The identified real flux is given as follows:

$$
\widehat{\psi}_{m}=\sqrt{\left(\widehat{\psi}_{m d}\right)^{2}+\left(\widehat{\psi}_{m q}\right)^{2}}
$$

The accurate estimation of rotor position and speed in the flux-weakening area is obtained from (17) and (19) by using identified flux $\widehat{\psi}_{m}$. From (30)-(32), EKF for magnetic flux identification is a fourth-order linear system. After choosing $\left[\begin{array}{llll}i_{d} & i_{q} & \psi_{m d} & \psi_{m q}\end{array}\right]^{T}$ as state variable and $\left[\begin{array}{ll}i_{d} & i_{q}\end{array}\right]^{T}$ as output,

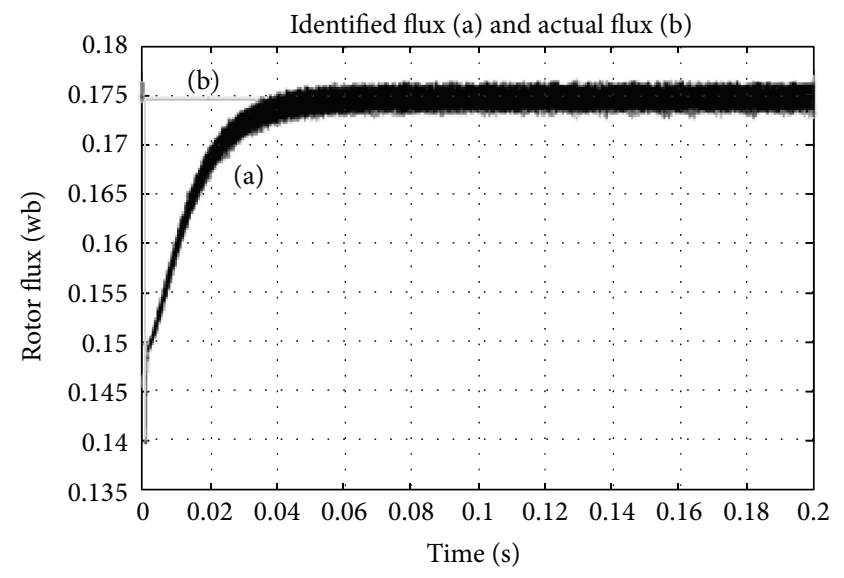

Figure 6: Wave of assumed flux and identified flux.

the proposed magnetic flux identification system is linear to the input and output.

In this part, SPMSM mathematical model based on EKF is induced, and the permanent magnet flux of SPMSM is identified by recurrent operation of EKF. The influence on uncertainties in magnetic magnet flux is eliminated from the position estimation.

\section{System Control Structure}

The simulation block of sensorless SPMSM drive system by using the proposed position/speed estimation and EKF based permanent magnet flux identification is shown in Figure 3. The rotor-flux-oriented vector control is employed as the basic control strategy for SPMSM drive. The reference voltage vectors are obtained by current proportional integral (PI) regulators. As shown in Figure 3, "space vector pulsewidth modulation (SVPWM)" module realizes SVPWM or overmodulation algorithm according to the amplitude of the reference voltage vector. "Sampling" module detects only 3phase motor voltage for estimating the position and speed of 


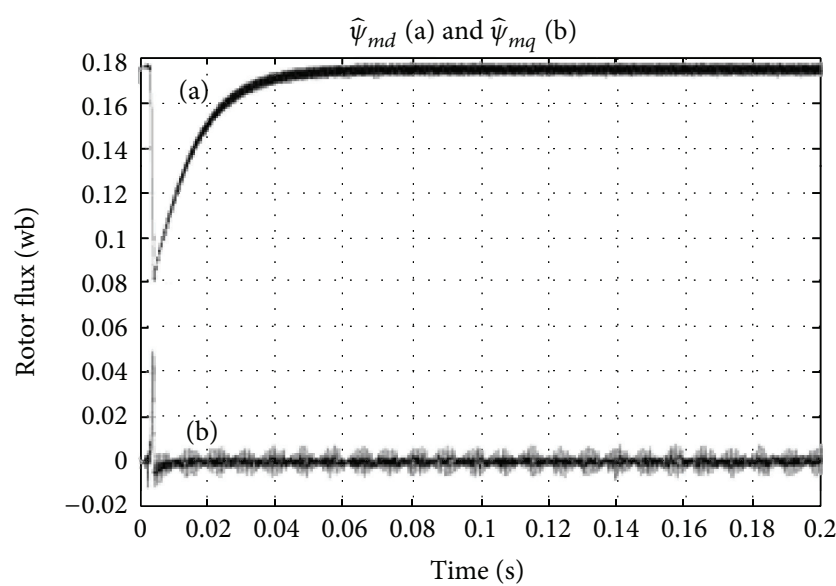

FIgURE 7: Rotor flux in $d-q$ frame.

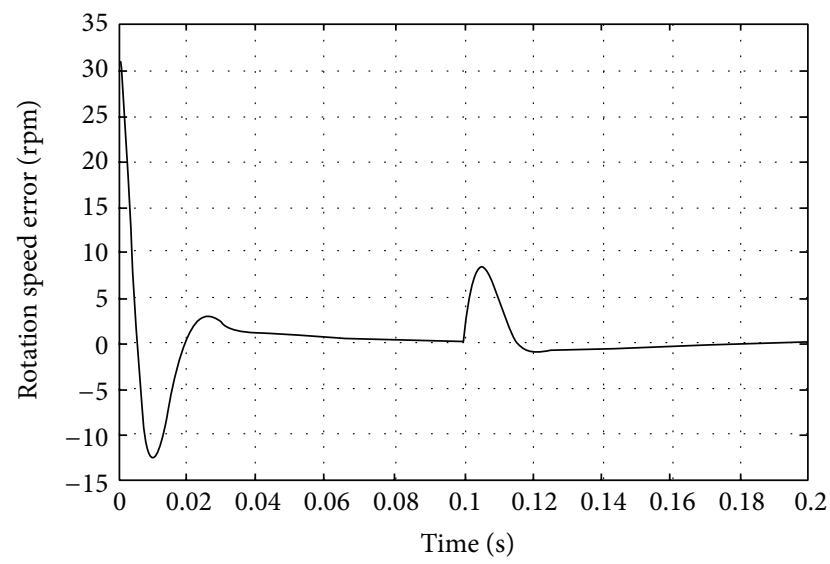

FIgURE 8: Speed estimation error with load torque from $2 \mathrm{Nm}$ to $4 \mathrm{Nm}$.

sensorless control. "Position estimation, flux identification" module identifies the permanent magnet flux by EKF and estimates the position and speed by the proposed estimation method. "Flux identification using EKF" module identifies the permanent magnet flux, whose result $\left(\widehat{\psi}_{m}\right)$ is sent to "position estimation" module (17) for rotor position estimation. In this system, the rotor position estimation by using the combination of motor complex number model based position estimation and position estimation error suppression PI controller and the permanent magnet flux identification by EKF are very interrelated to each other. The $d$ - $q$ axis reference voltages, currents, and estimated speed are used in flux identification. The detailed contents of "flux identification using EKF" block are shown in Figure 4.

\section{Simulation Study}

In order to verify the proposed control strategy, a model of pulse width modulation (PWM) inverter-fed SPMSM drive system is built in MATLAB9.0/SIMULINK platform. Simulation studies are carried out with a $10 \mathrm{kHz}$ control frequency. The parameters of SPMSM used in the simulation are listed in Table 1.

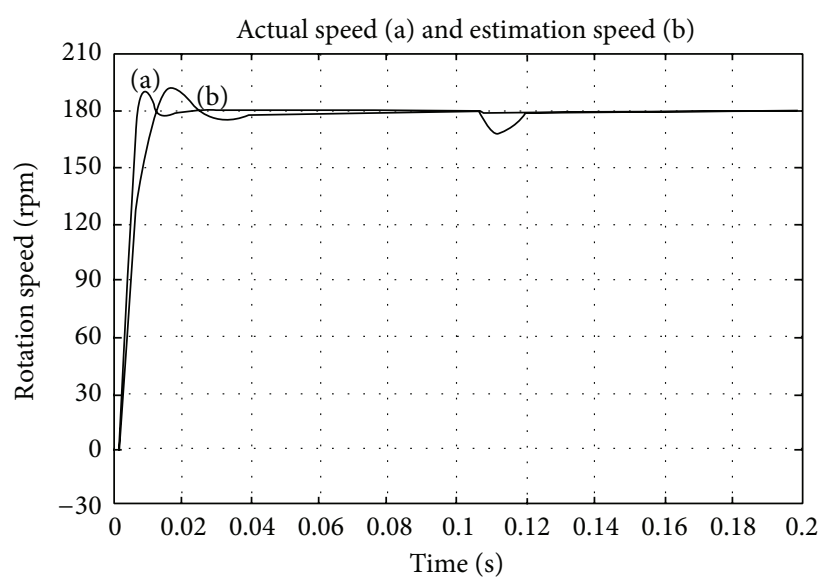

FIgURE 9: Speed estimation with load torque from $2 \mathrm{Nm}$ to $4 \mathrm{Nm}$.

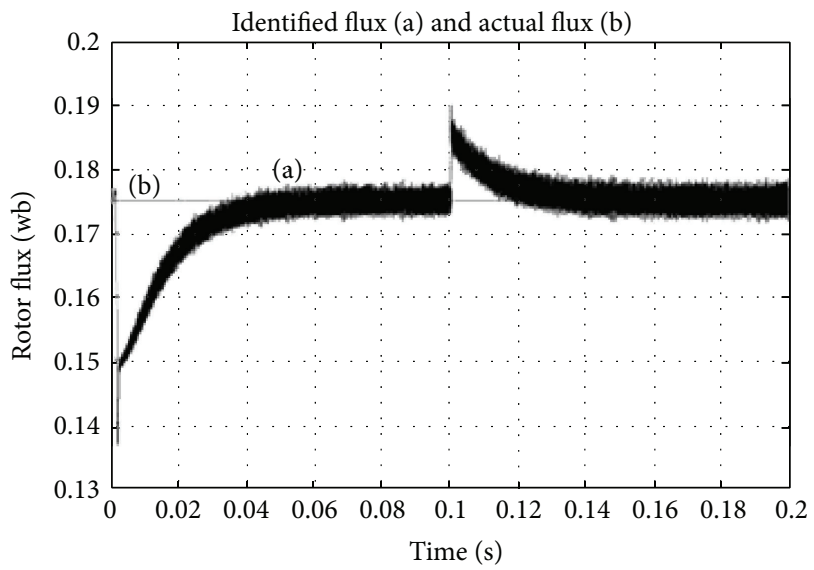

FIgURE 10: Flux identification with load torque from $2 \mathrm{Nm}$ to $4 \mathrm{Nm}$.

TABLE 1: Parameters for the motor and inverter systems in simulations.

\begin{tabular}{lc}
\hline Parameters & Value \\
\hline$d$ axis inductance $L_{S}$ & $8.5 e^{-3} \mathrm{H}$ \\
Stator resistance $R_{S}$ & $2.875 \Omega$ \\
Back-EMF coefficient $K_{E}$ & $0.2256 \mathrm{~V} /(\mathrm{rad} / \mathrm{s})$ \\
Pole pairs $p$ & 4 \\
DC link voltage $\mathrm{V}_{\mathrm{dc}}$ & $540 \mathrm{~V}$ \\
Load torque $T_{L}$ & $10 \mathrm{Nm}$ \\
Maximum allowable current & $10 \mathrm{~A}$ \\
of motor and inverter $I_{q \max }$ & $0.8 e^{-3} \mathrm{Nm}{ }^{2}$ \\
Inertia moment $l$ & $0.175 \mathrm{wb}$ \\
Magnet flux linkage $\psi_{m}$ & $1000 \mathrm{rpm}$ \\
Motor rated speed $\omega$ &
\end{tabular}

Figure 5 shows the error between the estimated position by proposed estimation method and the real position from position sensor under no load condition. It is clear that the real position is consistent with the estimated position which is obtained by the proposed estimation method when 


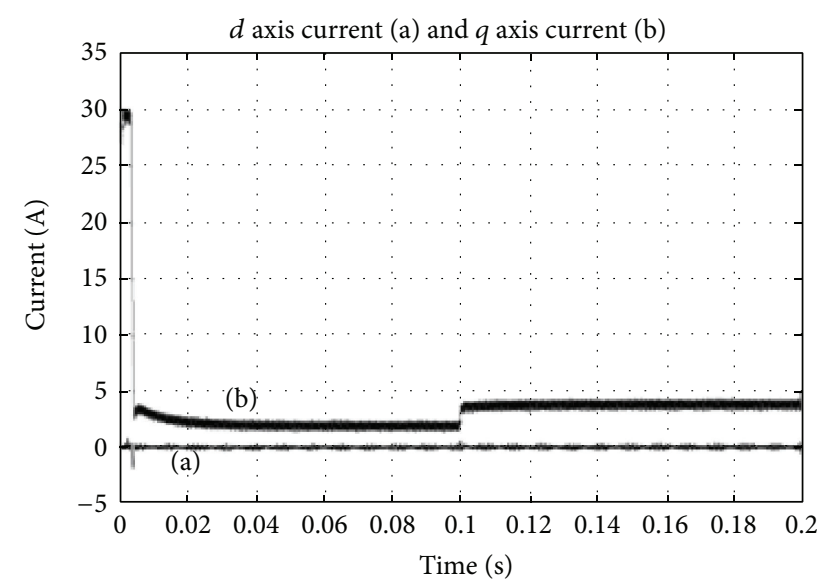

FigURE 11: $d-q$ frame currents with load torque from $2 \mathrm{Nm}$ to $4 \mathrm{Nm}$.

the motor speed comes to steady state. Figure 6 shows that the real flux and the flux that is identified by EKF are well matched. Figure 7 shows the $d$ axis and $q$ axis values of the identified flux $\widehat{\psi}_{m}$. We know that $\psi_{m d}$ converges to $\widehat{\psi}_{m}$ and $\psi_{m q}$ converges to 0 . It is clear that the proposed permanent magnet flux identification scheme is correct.

As mentioned above, the SPMSM drive system that includes permanent magnet flux identification by EKF exhibits a very good steady-state performance. Simulations are conducted to examine the dynamic performance of the proposed identification scheme in the case of load torque change. Figure 8 shows the estimation error of rotor speed (the error between the estimated speed and the real speed from the rotor position sensor) and Figure 9 shows the estimated speed under the condition of load torque change. Figure 10 shows the real flux and the identified flux when the load torque is changed. Figure 11 shows the $d-q$ axis current curves.

Figure 12 shows the speed estimation curves obtained from the back-EMF based estimator $[6,11]$ and our proposed estimator. The motor is accelerated from speed 0 to speed $1200 \mathrm{r} / \mathrm{min}$. Estimation time is about $0.28 \mathrm{~s}$ in the case of using back-EMF based method and is about $0.25 \mathrm{~s}$ in the case of applying our proposed method. In the steady state, vibrations are about $2 \%$ when back-EMF based estimator is used and are about $0.4 \%$ when our method is used. The performance of our proposed method is quite similar to the reference curve compared to back-EMF based method. Figure 13 shows the assumed flux and identified flux in the demagnetization state. Figure 14 shows the position estimation error curves obtained from the back-EMF based estimator $[6,11]$ and our proposed estimator. The position estimation error generated from the proposed position estimation method is reduced to $25 \%$ with respect to when the back-EMF based method is used. The simulation results show that both estimating the rotor position and speed by our proposed method and identifying the flux by EKF are done effectively.

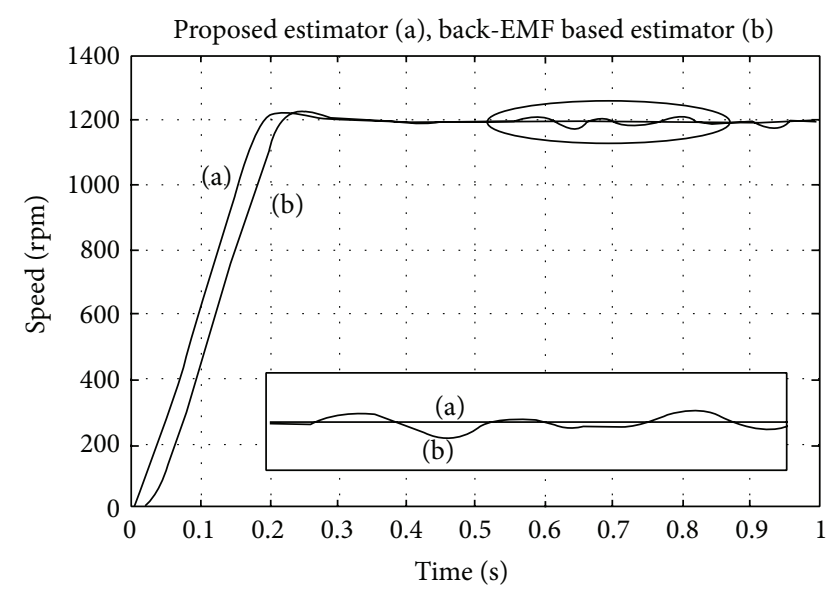

FIGURE 12: Estimation response of step speed command.

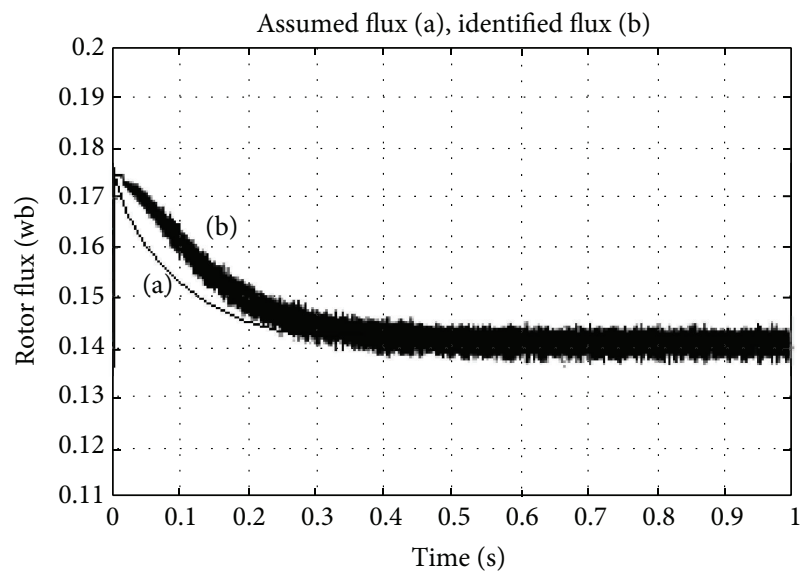

FIGURE 13: Identified flux in the demagnetization state.

\section{Conclusion}

In this paper, the rotor position angle is estimated by a novel position estimator based on complex number model of SPMSM. By using an error suppression PI controller that is combined with imaginary part of estimator, the estimation error of rotor position angle caused by the uncertainty of system parameters is converged to zero quickly. By identifying the permanent magnet flux by EKF, the sensitivity to uncertainty of permanent magnet flux that may be generated in the flux-weakening region is eliminated. Simulation results demonstrated that both the estimation of the rotor position and the accurate identification of permanent magnet flux are realized with satisfying performance. The estimation error of rotor position is limited within $0.4 \%$, and the identification error of permanent magnet flux is realized with $0.8 \%$. The step response rate has decreased about $10 \%$ and the position estimation error is reduced to $25 \%$ compared to using the back-EMF based position estimation method under the same control condition. 


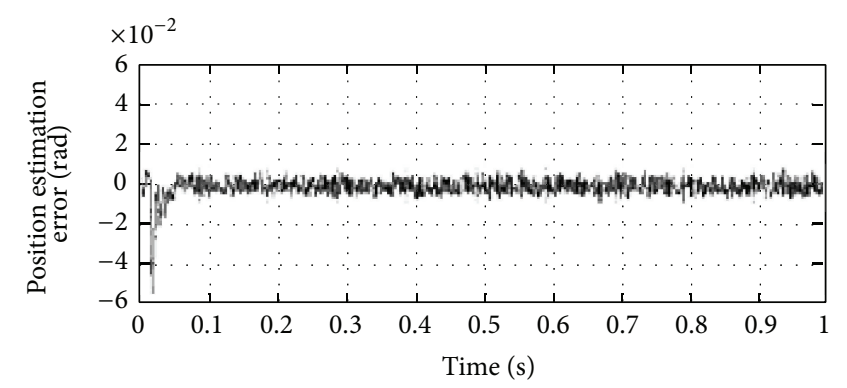

(a)

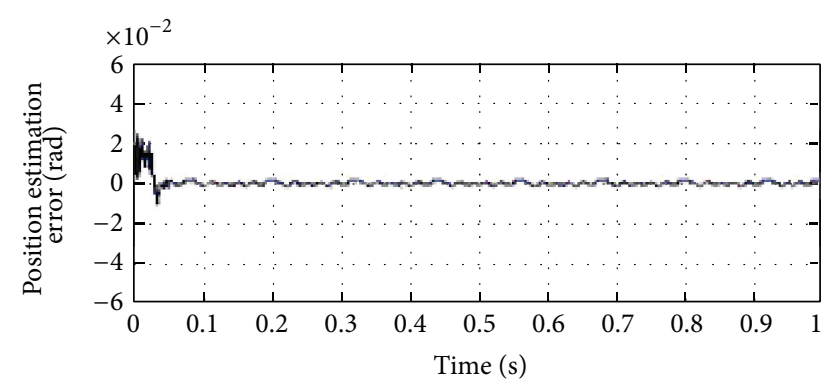

(b)

Figure 14: Position estimation error. (a) Back-EMF based estimator, (b) proposed estimator.

\section{Conflict of Interests}

The authors declare that there is no conflict of interests regarding the publication of this paper.

\section{Acknowledgments}

This work was supported by the National Natural Science Foundation of China under Grants 61074073 and 61034005, the Fundamental Research Funds for the Central Universities (Grant nos. N130504002 and N130104001), and Synthetical Automation for Process Industries (SAPI) Fundamental Research Funds 2013ZCX01-07.

\section{References}

[1] N. P. Quang and J. A. Dittrich, Vector Control of Three-Phase AC Machine, Springer, Berlin, Germany, 2008.

[2] Z. H. Liu, S. W. Zhou, K. Liu, and J. Zhangg, "Permanent magnet synchronous motor multiple parameter identification and temperature monitoring based on binary-modal adaptive wavelet particle swarm optimization," Acta Automatica Sinica, vol. 39, no. 12, pp. 2121-2130, 2013.

[3] Y. Kung and N. T. Hieu, "Simulink/ModelSim co-simulation of sensorless PMSM speed controller using reduced-order extended Kalman filter," in Proceeding of the 21st IEEE International Symposium on Industrial Electronics (ISIE '12), pp. 14051410, Hangzhou, China, May 2012.

[4] M. C. Huang, A. J. Moses, and F. Anayi, "The comparison of sensorless estimation techniques for PMSM between extended Kalman filter and flux-linkage observer," in Proceedings of the 21st Annual IEEE Applied Power Electronics Conference and Exposition (APEC '06), pp. 654-659, Dallas, Tex, USA, March 2006.

[5] X. Xiao, M. Zhang, Y. Li, and M. Li, "On-line estimation of permanent magnet flux linkage ripple for PMSM based on a Kalman filter," in Proceedings of the 32nd Annual Conference on IEEE Industrial Electronics (IECON '06), pp. 1171-1175, Paris, France, November 2006.

[6] F. Genduso, R. Miceli, C. Rando, and G. R. Galluzzo, "Back EMF sensorless-control algorithm for high-dynamic performance PMSM," IEEE Transactions on Industrial Electronics, vol. 57, no. 6, pp. 2092-2100, 2010.

[7] X. C. Liu, C. H. Song, and E. H. Chu, "Partial variable stabilization of a class of uncertain systems with uncertain equilibrium point," Acta Automatica Sinica, vol. 39, no. 6, pp. 780-789, 2013.

[8] B. Nahid-Mobarakeh, F. Meibody-Tabar, and F.-M. Sargos, "Mechanical sensorless control of PMSM with online estimation of stator resistance," IEEE Transactions on Industry Applications, vol. 40, no. 2, pp. 457-471, 2004.

[9] M. Comanescu and T. D. Batzel, "Full order EMF observer for PMSM-design, analysis and performance under improper speed signal," in Proceedings of the 4th International Systems Conference (SysCon '10), pp. 86-90, April 2010.

[10] Y. C. Shi, K. Sun, H. Y. Ma, and L. P. Huang, "Permanent magnet flux identification of IPMSM based on EKF with speed sensorless control," in Proceeding of the 36th Annual Conference of the IEEE Industrial Electronics Society (IECON '10), pp. 22522257, Glendale, Ariz, USA, November 2010.

[11] M. N. Eskander, O. M. Arafa, and O. A. Mahgoub, "Sensorless control of PMSM and BDCM based on EMF extraction and extended kalman estimator," in International Symposium on Industrial Electronics (ISIE '06), pp. 2168-2175, July 2006.

[12] M. Wu, R. Zhao, and J. Wang, "Sensorless estimation and convergence analysis based on MRAS for PMSM," in Proceedings of the 8th World Congress on Intelligent Control and Automation (WCICA '10), pp. 1641-1644, Jinan, China, July 2010.

[13] X. Xiao, Y. Li, M. Zhang, and Y. Liang, "A sensorless control based on MRAS method in interior permanent-magnet machine drive," in Proceedings of the International Conference on Power Electronics and Drive Systems (PEDS '05), vol. 1, pp. 734-738, Kuala Lumpur, Malaysia, December 2005.

[14] Y. Liang and Y. Li, "Sensorless control of PM synchronous motors based on MRAS method and initial position estimation," in Proceedings of the 6th International Conference on Electrical Machines and Systems (ICEMS '03), vol. 1, pp. 96-99, Beijing, China, November 2003.

[15] T. Orlowska-Kowalska and M. Dybkowski, "Stator-currentbased MRAS estimator for a wide range speed-sensorless induction-motor drive," IEEE Transactions on Industrial Electronics, vol. 57, no. 4, pp. 1296-1308, 2010.

[16] Y. Shi, K. Sun, L. Huang, and Y. Li, "Online identification of permanent magnet flux based on extended Kalman filter for IPMSM drive with position sensorless control," IEEE Transactions on Industrial Electronics, vol. 59, no. 11, pp. 4169-4178, 2012.

[17] A. Arias, X. Rain, and M. Hilairet, "Enhancing the flux estimation based sensorless speed control for switched reluctance machines," Electric Power Systems Research, vol. 104, pp. 62-70, 2013.

[18] T. D. Batzel and K. Y. Lee, "An approach to sensorless operation of the permanent-magnet synchronous motor using diagonally 
recurrent neural networks," IEEE Transactions on Energy Conversion, vol. 18, no. 1, pp. 100-106, 2003.

[19] F. M. Fayez, "Intelligent optimal recurrent wavelet elman neural network control system for permanent-magnet synchronous motor servo drive," IEEE Transactions on Industrial Informatics, vol. 9, no. 4, pp. 1986-2003, 2013.

[20] S. H. Fang, J. Y. Chen, M. D. Shieh, and J. S. Lin, "Subspacebased blind channel estimation by separating real and imaginary symbols for cyclic-prefixed single-carrier systems," IEEE Transactions on Broadcasting, vol. 59, no. 4, pp. 698-704, 2013.

[21] N. Ito, N. Ono, E. Vincent, and S. Sagayama, "Designing the wiener post-filter for diffuse noise suppression using imaginary parts of inter-channel cross-spectra," in IEEE International Conference on Acoustics, Speech, and Signal Processing (ICASSP '10), pp. 2818-2821, Dallas, Tex, USA, March 2010.

[22] J. S. Park, S. M. Jung, H. W. Kim, and M. J. Youn, “Design and analysis of position estimator based on instantaneous power for sensorless drive of permanent magnet synchronous motor," in Proceedings of the 8th International Conference on Power Electronics and ECCE Asia (ICPE ECCE '11), pp. 1140-1147, Jeju, Republic of Korea, June 2011.

[23] I. R. Maria, Kalman and Extended Kalman Filters: Concept, Derivation and Properties, Rovisco Pais, 2004.

[24] S. V. Sagar and K. D. Joseph, "Speed estimation algorithms for sensorless control of PMSM," in Proceedings of the IEEE International Multi Conference on Automation, Computing, Control, Communication and Compressed Sensing (iMac4s '13), pp. 138-143, Kottayam, India, February 2013.

[25] M. G. Shady, G. Damian, and W. F. John, "Stator current model reference adaptive systems speed estimator for regeneratingmode low-speed operation of sensorless induction motor drives," IET Electric Power Applications, vol. 7, no. 7, pp. 597606, 2012.

[26] C. Park and W. Kwon, "Simple and robust speed sensorless vector control of induction motor using stator current based MRAC," Electric Power Systems Research, vol. 71, no. 3, pp. 257266, 2004.

[27] M. S. Zaky, M. Khater, H. Yasin, and S. S. Shokralla, "Very low speed and zero speed estimations of sensorless induction motor drives," Electric Power Systems Research, vol. 80, no. 2, pp. 143151, 2010.

[28] Y. Zhao, W. Qiao, and L. Wu, "An adaptive quasi-sliding-mode rotor position observer-based sensorless control for interior permanent magnet synchronous machines," IEEE Transactions on Power Electronics, vol. 28, no. 12, pp. 5618-5629, 2013. 


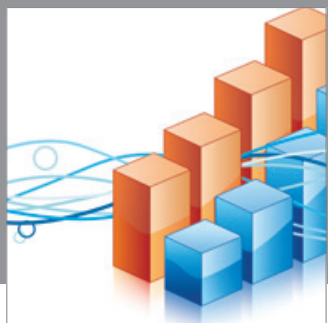

Advances in

Operations Research

mansans

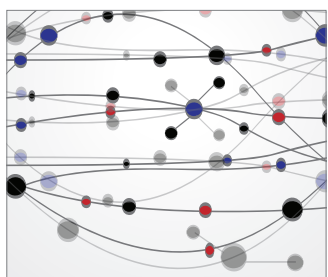

The Scientific World Journal
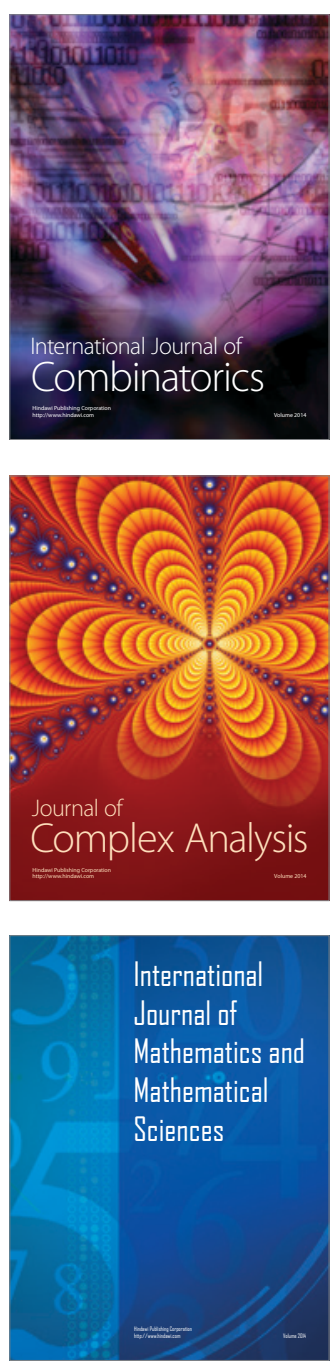
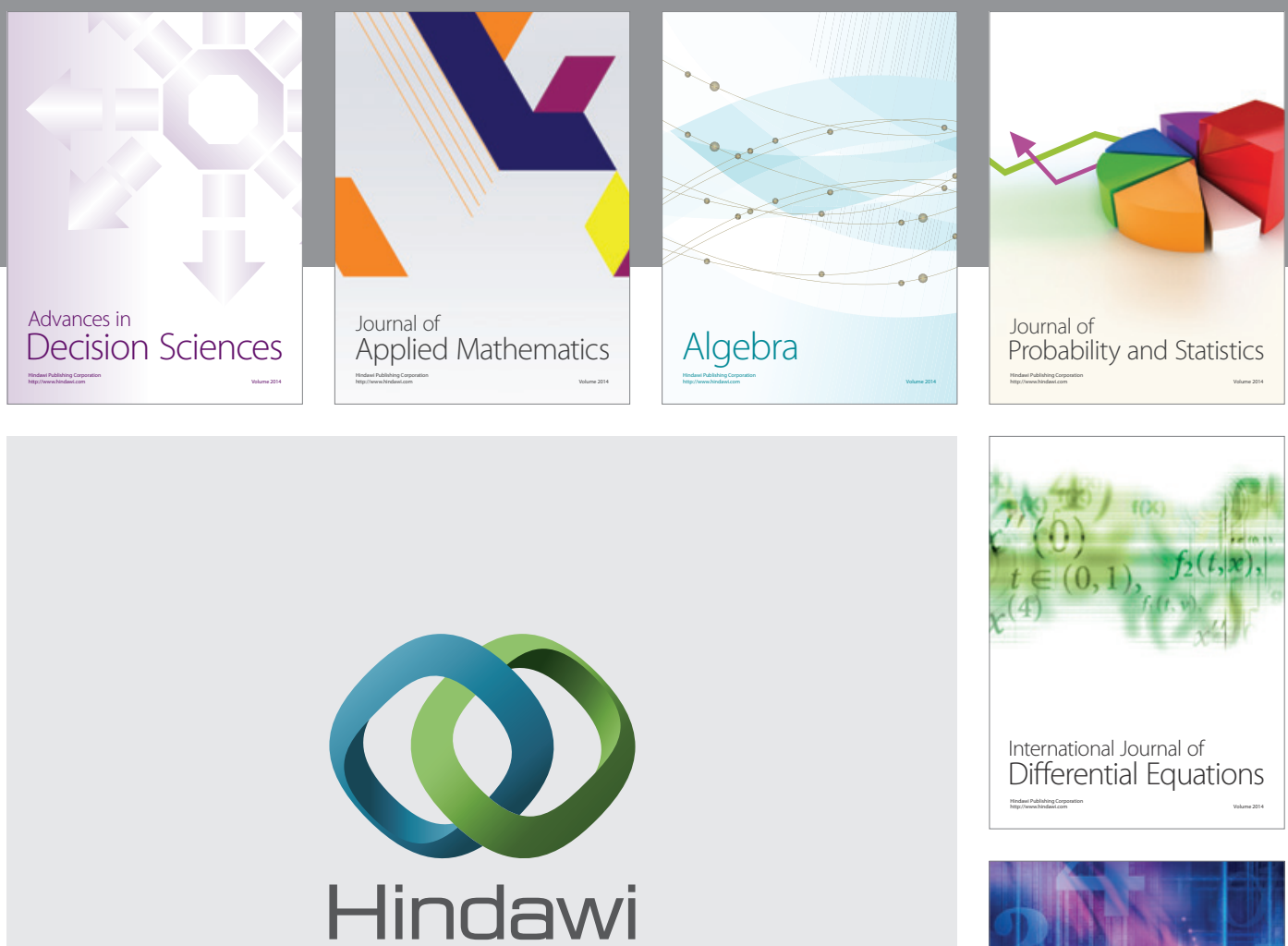

Submit your manuscripts at http://www.hindawi.com
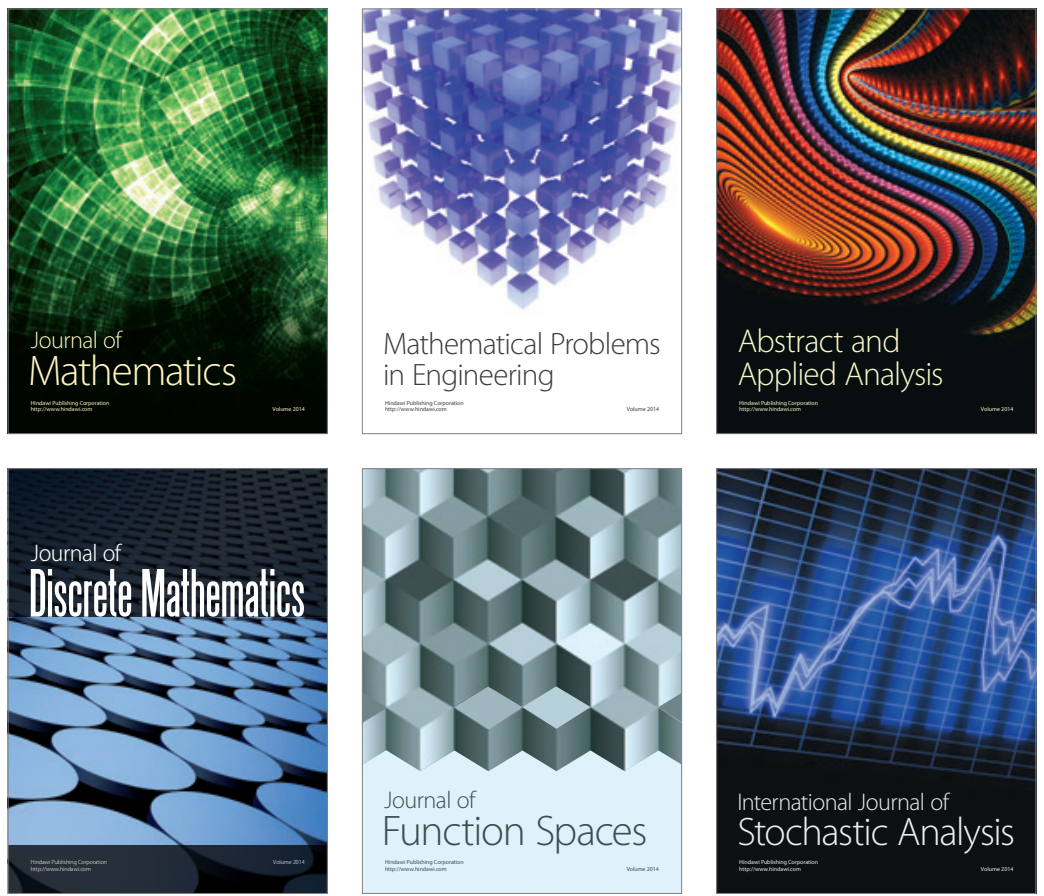

Journal of

Function Spaces

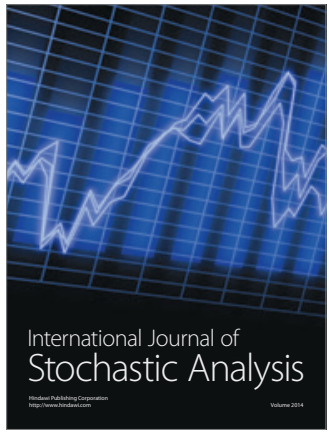

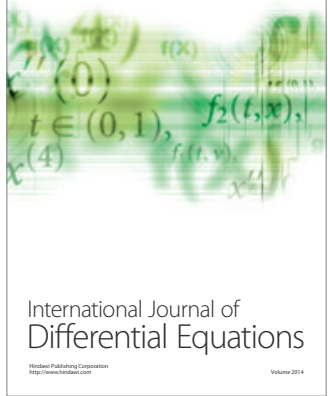
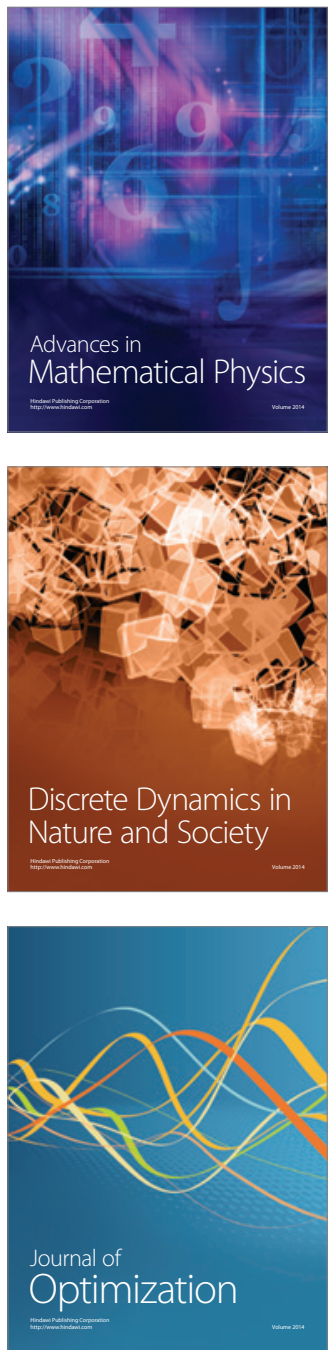